\title{
Halloysite nanotubes filled with salicylic acid and sodium diclofenac: effects of vacuum pumping on loading and release properties
}

\author{
Lorenzo Lisuzzo $^{1}$ - Giuseppe Cavallaro ${ }^{1,2}$ (D) Stefana Milioto ${ }^{1,2} \cdot$ Giuseppe Lazzara $^{1,2}$
}

Received: 15 October 2020 / Accepted: 15 January 2021 / Published online: 2 February 2021

(c) The Author(s) 2021

\begin{abstract}
In this work, we investigated the effects of the vacuum pumping on both the loading efficiencies and the release kinetics of halloysite nanotubes filled with drug molecules dissolved in ethanol. As model drugs, salicylic acid and sodium diclofenac were selected. For comparison, the loading of the drug molecules was conducted on platy kaolinite to explore the key role of the hollow tubular morphology on the filling mechanism of halloysite. The effects of the pressure conditions used in the loading protocol were interpreted and discussed on the basis of the thermodynamic results provided by Knudsen thermogravimetry, which demonstrated the ethanol confinement inside the halloysite cavity. Several techniques (TEM, FTIR spectroscopy, DLS and $\zeta$-potential experiments) were employed to characterize the drug filled nanoclays. Besides, release kinetics of the drugs were studied and interpreted according to the loading mechanism. This work represents a further step for the development of nanotubular carriers with tunable release feature based on the loading protocol and drug localization into the carrier.
\end{abstract}

\section{Graphic abstract}

The filling efficiency of halloysite nanotubes is enhanced by the reduction of the pressure conditions used in the loading protocol.
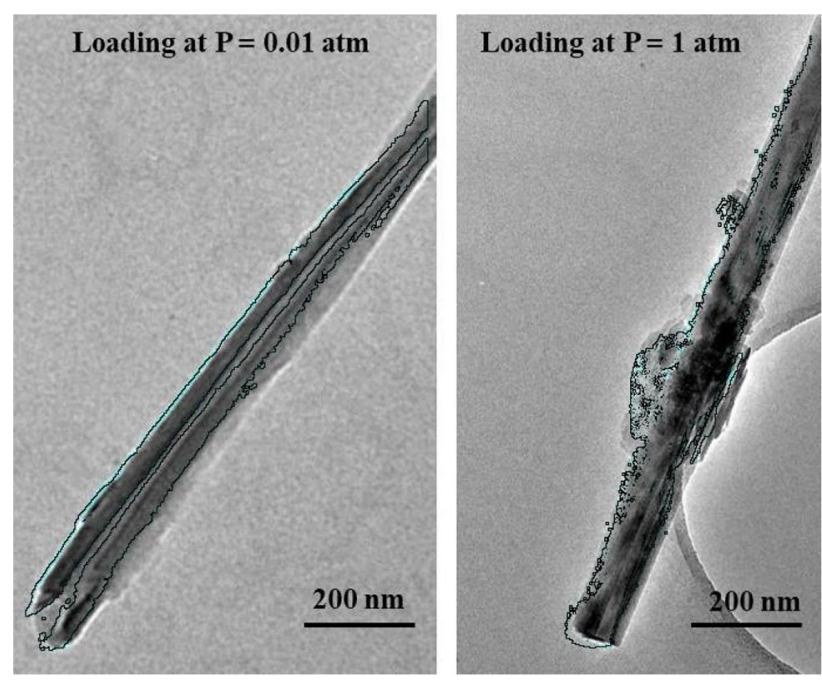

Keywords Halloysite nanotubes · Sustained release · Clay nanoparticles · Drug loading · Knudsen thermogravimetry

Giuseppe Cavallaro

giuseppe.cavallaro@unipa.it

Extended author information available on the last page of the article 


\section{Introduction}

In the very last decades, we observed a growing interest of the scientific community in the study of clays, which were employed in numerous applications. The reason for their rapid diffusion has to be found in their wide variety of different and tunable features, including the morphology, size, interfacial chemistry [1-7]. Moreover, they are natural occurring building blocks that can be used as raw components for the design of new green and eco-sustainable materials [8-13]. Nowadays, the latter aspect appears to be the most important and attractive factor since it deals with some environmental needs which demand great efforts especially from researchers and technologists [14-16]. Halloysite is a very promising aluminosilicate whose unit formula is $\mathrm{Al}_{2} \mathrm{Si}_{2} \mathrm{O}_{5}(\mathrm{OH})_{4} \cdot 2 \mathrm{H}_{2} \mathrm{O}$ and whose most intriguing feature is its distinctive shape $[17,18]$. The dimensions of halloysite nanotubes (HNTs) are influenced by their natural geological deposit [19]. Compared to the carbon nanotubes (CNTs), halloysite nanotubes are easily available and abundant worldwide. Consequently, the price of HNTs is more than 100 times lower with respect to that of CNTs. Moreover, HNTs are biocompatible nanomaterials, while CNTs can be toxic to the human health reducing their potential applications in biotechnological and pharmaceutical fields. Based on the European Union Standards, the amount of heavy metals in halloysite is much lower than the maximum limits on harmful species [1]. Similar to carbon nanotubes, HNTs can be easily rendered conductive by metallization and/or coating with polymers $[20,21]$. In addition, both HNTs and CNTs are effective fillers for the mechanical reinforcement of polymeric matrix driving to the preparation of smart nanocomposites [20]. According to the previous considerations, HNTs are considered a green alternative nanofiller with a broad range of technological applications. Basically, the nanotubes length is from $200 \mathrm{~nm}$ to $2 \mu \mathrm{m}$, their internal diameter is $15-50 \mathrm{~nm}$, whereas the external diameter is 50-200 nm [22]. Furthermore, outer and internal surfaces possess opposite charges because of their different chemistry (Al-OH and $\mathrm{Si}-\mathrm{O}-\mathrm{Si}$ groups, respectively) [23]. Thus, the inner ( $\mathrm{Al})$ surface is positively charged, while the outer $(\mathrm{Si})$ surface is negatively charged [24]. Consequently, this unique property can be exploited for the surface modification with organic molecules (e.g., polymers, biopolymers or surfactants), through both electrostatic and van der Waals interactions, thus leading to the design of hybrid and bio-hybrid systems that find applications in a broad range of applications [25-34]. In general, electrostatic interactions are convenient to target the interaction/adsorption site for halloysite nanotubes [35-38]. Most interestingly, halloysite nanotubes can be loaded with negatively charged active species, such as biological molecules, anti-corrosion compounds and antioxidants that can be protected inside the inner lumen of the nanotubes [39-41]. This one is a very important property, which endows the preparation of new smart nanocontainers for loading, storage and sustained release of chemical agents [42-45]. Moreover, halloysite exhibits low toxicity $[32,46,47]$. To the light of these aspects, thermo-responsive nanocarriers were prepared by the functionalization of HNTs with charged PNIPAAMs for the controlled delivery of drugs into biological systems [48]. Additionally, the design of new hybrid and biohybrid materials was carried out using drug-loaded halloysite for health applications as antimicrobial patches or anti-inflammatory drugs-releasing tablets [49-52]. Similarly, the nanotubes were used for the encapsulation of corrosion inhibitors with the aim to coat and protect metal surfaces [53-55]. Furthermore, HNTs were loaded with antioxidant species to preserve food by designing packaging nanomaterials [56] and, finally, clay nanotubes were used for cultural heritage treatment by loading anti-acid species [57]. Recent reviews and research articles evidenced that halloysite is a support for the development of antimicrobial nanocomposites [58-62]. The control of the loading mechanism is crucial to extend the HNTs applications as drug delivery systems and nanocontainers [63]. In this regard, the confinement effect of a liquid into a very narrow space inside of the nanoparticles plays a crucial role [64]. Similar observations can be considered for halloysite nanotubes. First, Price et al. proved that by subjecting the drug/clay dispersion to a vacuum pumping step, where the pressure is lower than the standard atmospheric value, the drug loading efficiency is greatly enhanced for comparison with encapsulation protocols carried out without any particular pressure control [65]. Hence, in our previous study, we demonstrated the confinement of water within the inner lumen of halloysite and this finding was shown to be deeply correlated with the driving mechanism of nanotubes filling and to the optimization of the loading efficiency. Accordingly, the vacuum pumping allows the overall pressure to approach the vapor pressure of the solvent, which evaporates faster, thus leaving empty space to be filled by the guest molecules that are forced to enter and gather inside [66]. Literature reports several studies on the loading of halloysite from organic solvents by using the vacuum assisted procedure [43, $67,68]$. Nevertheless, the physico-chemical insights of this mechanism have not been studied. In this work, we moved forward by investigating the confinement effect of an organic solvent, namely ethanol, to understand its influence on the loading mechanism, on the drug localization within the clay and on the releasing kinetics. Indeed, this seems to be the most important aspect for all the applications we discussed above, concerning the use of halloysite as controlled and tunable delivery system. 


\section{Experimental}

\section{Materials}

Kaolinite (Kao), diclofenac sodium salt (DF) and absolute ethanol (99.8\%) are Sigma Aldrich products. Salicylic acid (SA) is from Fluka. Halloysite nanotubes (HNTs) are from I-Minerals Inc. mined in the geological deposit of Latah County with physico-chemical properties detailed elsewhere [22]. As reported elsewhere [22], the investigated halloysite possesses a basal spacing of $7.58 \AA$.

\section{Drug loading protocols}

The encapsulation of salicylic acid and diclofenac sodium salt within the halloysite cavity was performed by ethanol suspensions following two protocols. First, we prepared drug solutions in ethanol by magnetically stirring for $2 \mathrm{~h}$ at $20^{\circ} \mathrm{C}$. The drugs concentration was at the maximum of their solubility (saturated solutions). Halloysite was added keeping a HNTs/drug ratio of 2:1. The HNTs/drug suspensions were subjected to ultrasonication for $5 \mathrm{~min}$. Afterwards, the dispersions were divided into two aliquots following two different protocols: (i) the dispersion was transferred to a vacuum jar for $30 \mathrm{~min}$ allowing to reduce the pressure conditions $(P=0.01 \mathrm{~atm})$ and to remove ethanol. Then, the vacuum was broken for $10 \mathrm{~min}$. The cyclic vacuum pumping step was repeated three times. (ii) The dispersion was kept under stirring for $2 \mathrm{~h}$ at $P=1 \mathrm{~atm}$. In both the two protocols, room temperature conditions were considered.

The same procedure was repeated using kaolinite to investigate the influence of the vacuum conditions on the filling process by altering the nanoclay morphological features. It should be noted that both drugs are highly soluble in ethanol. In particular, the solubilities in ethanol of salicylic acid and sodium diclofenac are 2.35 [69] and $0.5202 \mathrm{~mol} \mathrm{~L}^{-1}$ [70], respectively. These values are significantly larger compared to the corresponding molar solubilities in water $(0.0159$ [69] and $0.0674 \mathrm{~mol} \mathrm{~L}^{-1}$ [70] for salicylic acid and sodium diclofenac, respectively). Accordingly, loading protocols based on ethanol dispersions could enhance the amounts of drugs encapsulated within the clay nanotubes with respect to those expected using aqueous suspensions. Furthermore, both ethanol and water are largely employed for the filling of halloysite nanotubes with guest molecules as evidenced by recent reviews [21, 47] and research articles [40, 49, 66].

\section{Drug release kinetics}

The study of the drug release kinetics was carried out via UV-VIS spectrophotometry. Since the main purpose is to target the molecules accumulation within HNTs and to study the crucial effects on the delivery properties, water was used as releasing medium. Therefore, $10 \mathrm{mg}$ of loaded nanoclays (HNTs or Kao) was placed into water and the drugs release was studied as reported elsewhere [49]. It should be pointed out that the absorbance values at 276 and $296 \mathrm{~nm}$ were considered for diclofenac sodium salt and salicylic acid, respectively. Releasing profiles were determined using the following equation [71]:

$C_{n}^{\prime}=C_{n}+\left(V / V_{0}\right) \cdot \sum_{i}\left(C_{i}\right)$,

where $C_{n}$ and $C^{\prime}{ }_{n}$ refer to the drug concentrations in water before and after the correction, respectively. On the other hand, $V$ is the volume for each measured aliquot, while $V_{0}$ represents the full volume for each sample. The same procedure was followed for the samples whose drug loading was performed using both the two protocols described in the previous paragraph. Drug release tests were conducted three times. As concerns the dissolution of pure diclofenac and salicylic acid, the whole amounts of pure drugs could be detected in solution already at the first experimental point.

\section{Methods}

A Q5000 IR apparatus (TA Instruments) was employed for thermogravimetry (TG). The measurements were performed under inert atmosphere using different $\mathrm{NO}_{2}$ flows for the balance and the sample (10 and $25 \mathrm{~cm}^{3} \mathrm{~min}^{-1}$, respectively). The samples were heated at $20{ }^{\circ} \mathrm{C} \mathrm{min}^{-1}$ from room temperature to $700{ }^{\circ} \mathrm{C}$. The apparatus was calibrated using the Curie temperature of standards [72]. The drug loading amounts were calculated by the quantitative analysis of TG curves as detailed in Supplementary Material [73]. Knudsen thermogravimetry (KTG) measurements were conducted at $30{ }^{\circ} \mathrm{C}$ using Knudsen cells with a hole (diameter of $20 \mu \mathrm{m}$ ) on their top surface. Ethanol dispersions of both nanoclays (concentration of 50 $\mathrm{wt} \%$ ) were investigated by KTG measurements to explore the solvent evaporation process. According to literature [66], ethanol activity coefficients were calculated. The evaporation rate can be expressed as:

$\mathrm{dm} / \mathrm{dt}=K_{F} \cdot\left(P_{\text {int }}-P_{\text {ext }}\right)$,

where $K_{F}$ is a constant related to the volatile gas, $P_{\text {int }}$ is the partial pressure of the solvent vapor inside the cell and $P_{\text {ext }}$ is the external pressure. It should be noted that $K_{F}$ refers to a given gas escaping through a Knudsen orifice with specific geometrical size and at a specified temperature. Based on the fundamentals of KTG, the thermodynamic activity of ethanol $\left(a_{\mathrm{EtOH}}\right)$ can be estimated by the following equation:

$(\mathrm{dm} / \mathrm{dt})_{n} /(\mathrm{dm} / \mathrm{dt})_{\mathrm{EtOH}} \approx\left(P_{\text {int }}\right)_{n} /\left(P_{\text {int }}\right)_{\mathrm{EtOH}}=a_{\mathrm{EtOH}}$, 
being $(\mathrm{dm} / \mathrm{dt})_{n}$ and $(\mathrm{dm} / \mathrm{dt})_{\mathrm{EtOH}}$ the mass loss rates for the nanoclay ethanol dispersion and pure ethanol, respectively.

A Zetasizer NANOZS (Malvern Instruments) instrument was used to conduct both dynamic light scattering (DLS) and $\zeta$-potential experiments, which were carried out at $25^{\circ} \mathrm{C}$. The analyses of DLS curves provided the apparent hydrodynamic radius $(\mathrm{Rh})$ of pure and loaded clay nanotubes, while $\zeta$-potential data were helpful to monitor the effect of the drug loading on the HNTs surface charge.

TEM micrographs were acquired with a Jeol JEM 2100 microscope operating at $200 \mathrm{kV}$. A drop of each dispersion has been deposited in the grid, which was dried prior to the acquisition of TEM images. A $3 \mathrm{~mm}$ nickel grid holey carbon coated (Taab) was selected for TEM analyses. It should be noted that no coating was applied to the samples. TEM images of both pure and loaded HNTs were analyzed using ImageJ software [74] for the determination of the corresponding averaged gray-scale profiles.

Kinetics of drug releases were studied by UV-Vis spectrophotometer (Specord S600 Analytik Jena).

Fourier transform infrared (FTIR) spectra were determined using a Frontier FTIR spectrometer (PerkinElmer) at $25^{\circ} \mathrm{C}$ in the wavenumber range between 500 and $4000 \mathrm{~cm}^{-1}$. $\mathrm{KBr}$ pellets were employed for FTIR analyses.

\section{Results and discussion}

\section{Drugs loading onto halloysite nanotubes: mechanism and structure}

The amounts of organic molecules loaded into the nanoclays were determined through thermogravimetry taking into account the equilibrium moisture contents of all materials (before and after the drug loading). The moisture content data are reported in Supplementary Material (Table 1S). Compared to those of pristine clay minerals, the thermogravimetric curves of the hybrid materials evidenced a significant mass loss due to the decomposition of the organic moiety at ca. 250 and $180{ }^{\circ} \mathrm{C}$ for DF and SA, respectively (Fig. 1S in Supplementary Material). The pressure conditions induced a relevant effect on the loading efficiency of HNTs. In particular, the reduced pressure enhanced the filling efficacy for HNTs (the loading increases by factors 4 and 6 for DF and SA, respectively); whilst for Kao, the dependence on the preparation condition is less crucial although the reduced pressure generated an increase up to a factor 2 in the loading amount (Fig. 1).

The successful loading of salicylic acid into the nanotubes was confirmed by FTIR spectroscopy, which evidenced the presence of the characteristic signals of the drug (such as vibrations of $\mathrm{C}=\mathrm{C}$ and $-\mathrm{COO}^{-}$groups at 1485 and $1582 \mathrm{~cm}^{-1}$, respectively [51]) in the HNTs/SA hybrids.

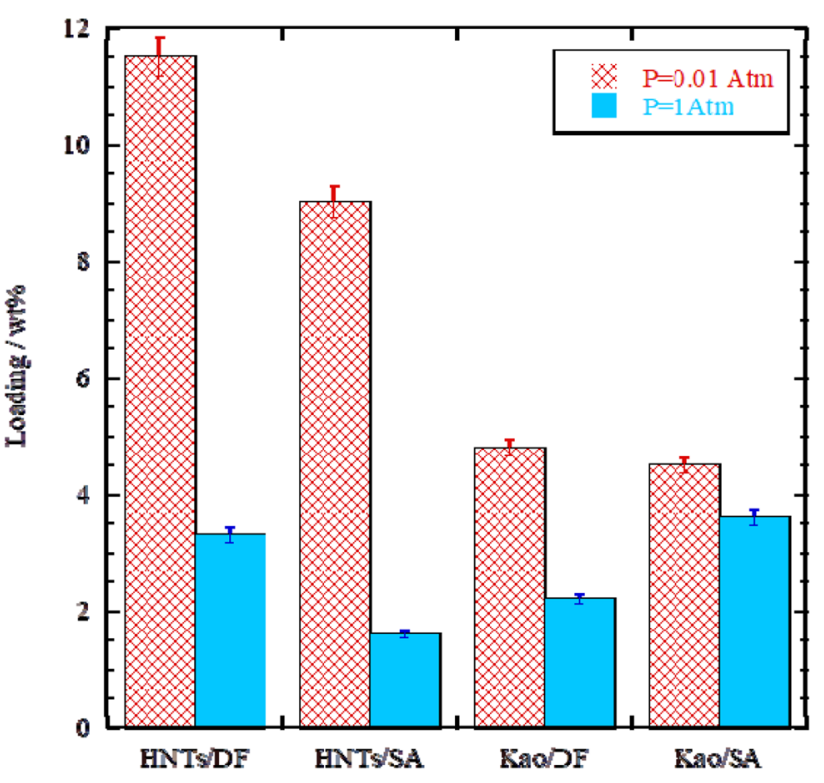

Fig. 1 Loading amounts for nanoclay/drug systems prepared at different pressure conditions

Morphological investigations were carried out by TEM on halloysite nanotubes before and after drugs loading under different pressure conditions. Figure 2 shows the images for pristine nanotubes as well as for HNTs/DF and HNTs/SA samples prepared with the different protocols. For a better visualization of the status of the HNTs cavity, an averaged gray-scale profile was plotted along the perpendicular direction of the main nanotube axis for all samples (see insets in Fig. 2) to have semi-quantitative additional insights about the effectiveness of the drug loading procedure. The empty cavity of ca. $10 \mathrm{~nm}$ with a wall thickness of $20 \mathrm{~nm}$ is evidenced by the gray-scale contrast. It is suggested that a lower pressure increases the filling of the halloysite lumen. The cavity appears full filled when the loading is conducted under reduced pressure for both DF and SA. These findings are a semi-qualitative proof of the TG data, which evidenced increases of the drug loading at $P=0.01 \mathrm{~atm}$. These aspects are in agreement with DLS measurements, since no change in the hydrodynamic radius can be observed after the loading of the drug inside the clay. As examples, the distribution curves of the intensity as a function of the hydrodynamic radius for HNTs and HNTs/DF samples are presented in Supplementary Material (Fig. 2S). The influence of the pressure on the loading efficiency and drug localization in halloysite nanotubes is correlated with the different volatility of confined and bulk solvents. Namely, the solvent into the lumen possesses a larger vapor pressure compared to the bulk one and, consequently, a flux of ethanol into the halloysite lumen is required to compensate the different evaporation velocity. Consequently, drug molecules 
Fig. 2 TEM images for HNT (a), HNT/drug prepared at $P=0.01 \mathrm{~atm}(\mathbf{b}, \mathrm{HNTs} / \mathrm{SA}$ and c, $\mathrm{HNTs} / \mathrm{DF}$ ) and at $P=1 \mathrm{~atm}$ (d, HNTs/SA and e, HNTs/DF)
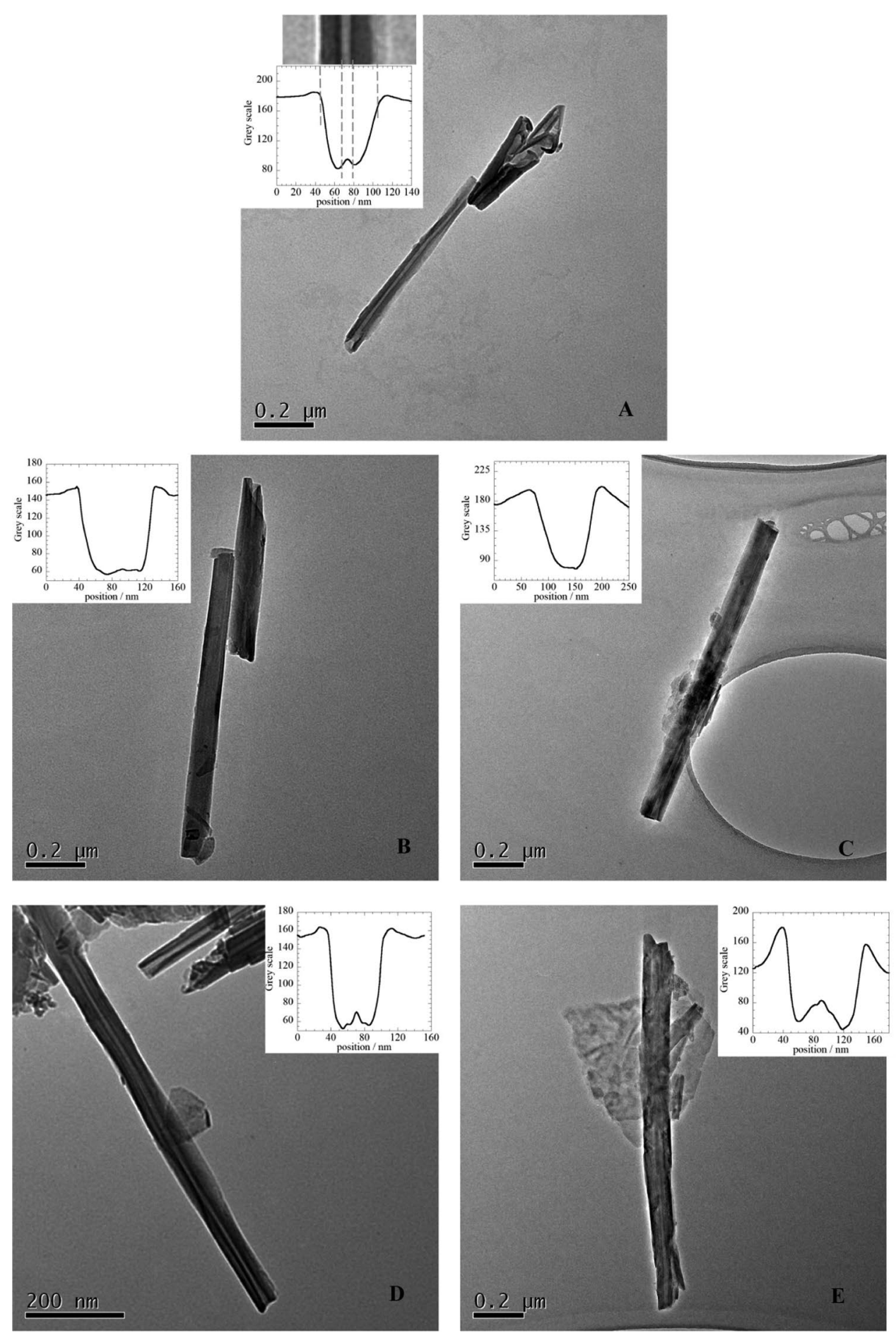

precipitate and their accumulation within the HNTs cavity can be observed.

Furthermore, the influence of the drugs loading on the HNTs surface charge was explored. To this purpose, $\zeta$-potential measurements were performed. As shown in Supplementary Material (Table 2S), the $\zeta$-potential of halloysite is not largely altered by the encapsulation of anionic drugs within the HNTs cavity. Contrary to these results, literature [21, 34,
48] reports that the loading of anionic species from aqueous suspensions generates substantial variations of the HNTs $\zeta$ -potential as a consequence of the electrostatic attractions with the halloysite inner surface, which is positively charged in a wide $\mathrm{pH}$ range. This discrepancy is related to the influence of the solvent polarity (and the dielectric constant) on the acid/base dissociation equilibria. In general, the dissociation constants in ethanol are lower than those in water 
and, consequently, the formation of ions occurs in a smaller extent. On this basis, HNTs/drugs electrostatic interactions are reduced in ethanol compared to those in water.

\section{Drugs release controlled by the loading protocol}

Halloysite nanotubes have been deeply investigated for applications in drug delivery. However, the possibility to target the drug localization within the nanomaterial is a promising result for attaining tunable release based on different preparation protocols, which would extend the applicability of these systems. On this basis, drug release experiments were performed for SA and DF embedded into the both nanoclays (halloysite and kaolinite) under the two different pressure conditions $(P=0.01 \mathrm{~atm}$ and $P=1 \mathrm{~atm})$. As a general result, the percentage of released drug showed a monotonic increase with the time reaching a constant value that corresponds to the quantitative release of the loaded material (Figs. 3, 4). These trends are typically observed for small molecules released by nanoclays and can be interpreted by considering the drug/nanomaterial interactions as well as the potential intercalation and/or localization of the active molecules in the porous support [75]. It was observed that the loading protocol generates relevant effects on the release profile for halloysite nanotubes based systems (Figs. 3, 4). In particular, the nanotubes loaded at reduced pressure showed slower release kinetics than the corresponding systems obtained at ambient pressure. These results reflect the drugs localization within the HNTs cavity. It should be noted

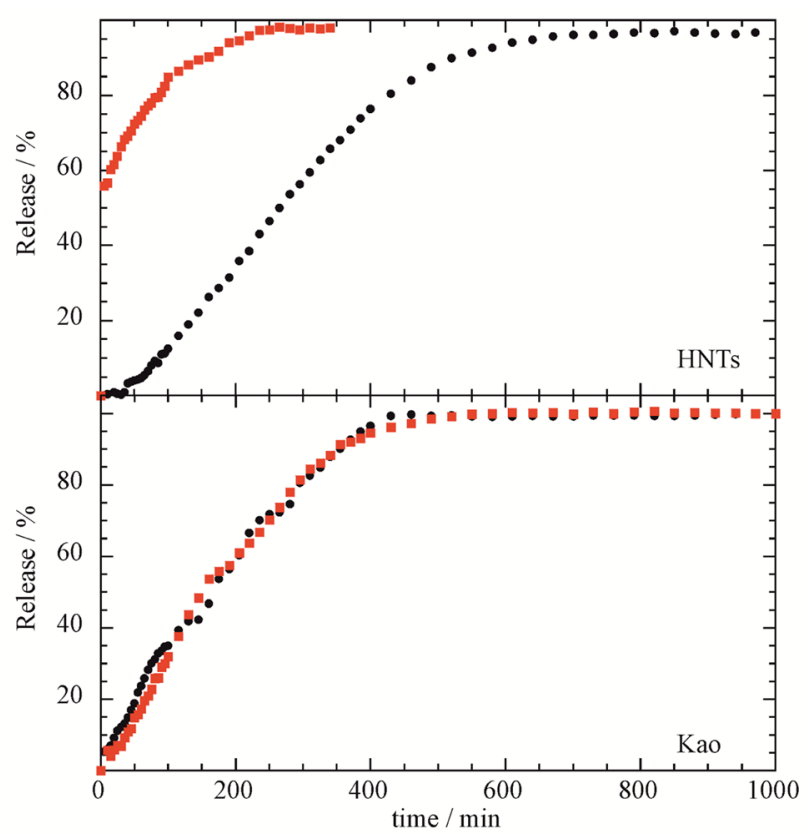

Fig. 3 Release kinetics of sodium diclofenac salt loaded at $P=1$ atm (red squares) and $P=0.01$ atm (black circles)

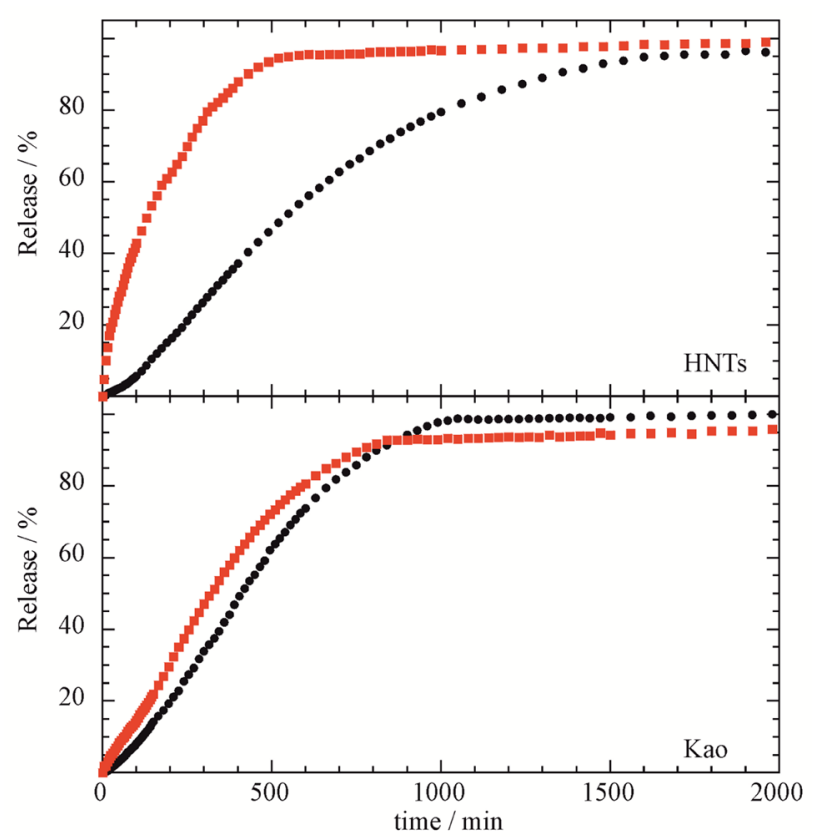

Fig. 4 Release kinetics of salicylic acid loaded at $P=1$ atm (red squares) and $P=0.01$ atm (black circles)

that the incorporation into the halloysite lumen generates a sustained release profile due to confinement and tortuosity effects that are less relevant when the drug is mainly outside the nanotubes. To validate this hypothesis, the release experiments for kaolinite/drug hybrids were considered. Interestingly, the release from kaolinite based systems was not influenced by the loading protocol (Figs. 3, 4). These results are in agreement with the morphological planar structure of kaolinite that, differently than halloysite, does not possess a cavity to be preferentially filled under reduced pressure conditions. For a clear quantitative evaluation, the time necessary for the release of $50 \%\left(t_{50 \%}\right)$ of the payload is reported in Table 1 for all the systems. It should be noted that the $t_{50 \%}$ values were calculated by considering the maxima of the UV-Vis absorbance as $100 \%$ of drug releases, which are in agreement with the loading results obtained from thermogravimetric analysis. Based on $t_{50 \%}$ values, one

Table 1 Drug release kinetics data

\begin{tabular}{lll}
\hline & \multicolumn{2}{l}{$t_{50 \%} / \mathrm{min}$} \\
\cline { 2 - 3 } & $P=0.01 \mathrm{~atm}$ & $P=1 \mathrm{~atm}$ \\
\hline HNTs & \\
DF & $270 \pm 30$ & $4.7 \pm 0.2$ \\
SA & $570 \pm 60$ & $135 \pm 3$ \\
Kaolinite & & \\
DF & $160 \pm 20$ & $163 \pm 12$ \\
SA & $425 \pm 20$ & $330 \pm 20$ \\
\hline
\end{tabular}


can state that the preparation protocol can tune the release time even by on order of magnitude in the case of halloysite nanotubes; whilst its effect is almost negligible for flat kaolinite clay mineral.

A general summary on the effect of the loading protocol is sketched in Fig. 5.

\section{Ethanol evaporation in clay nanostructures: mechanism for drugs loading}

The influence of the pressure conditions on the loading of anionic guest molecules was reported in aqueous media and it was strictly correlated with the water confinement [66]. Due to its confinement, water molecules exhibit a faster evaporation if they are located into the halloysite cavity. As a consequence, a flux of the solution driven by capillarity occurs within the HNTs cavity, in which the drug molecules accumulate once the water evaporation takes place. Such a process is certainly facilitated under low-pressure conditions due to the increased evaporation rate. It should be noted that the loading of anionic drugs in aqueous solvent can be favored by the electrostatic interactions. Namely, anionic compounds can interact with the HNTs inner surface, which possesses positive charges. On the contrary, the loading of anionic species in ethanol is slightly affected by electrostatic attractions as demonstrated by the $\zeta$-potential data of HNTs/ SA and HNTs/DF hybrids (see Table $2 \mathrm{~S}$ in Supplementary Material). Therefore, the loading mechanism by ethanol suspensions can be interpreted by considering the solvent confinement within halloysite cavity.

To verify this interpretation, the ethanol evaporation rate in the presence of halloysite nanotubes and platy kaolinite was explored by KTG experiments. The results are discussed in terms of relative vapor pressure after the normalization by the bulk ethanol evaporation $\left(P / P^{\circ}\right)$ as a function of the ethanol/clay mass ratio $\left(R_{\text {EtOH:Clay }}\right)$.

Figure 6 shows that $P / P^{\circ}>1$ was detected for halloysite suspensions with $R_{\text {EtOH:clay }}$ ranging between $6 \times 10^{-3}$ and $6 \times 10^{-2}$, while this peculiarity was not observed in kaolinite dispersions. On this basis, we can assert that a fraction of ethanol with a faster evaporation rate than that of pure solvent was induced by the presence of halloysite nanotubes. According to the Gibbs Thomson effect, the latter represents the thermodynamic proof of the ethanol confinement within the HNTs cavity. Based on geometric considerations and the bulk densities, halloysite nanotubes should be totally filled with ethanol for $R_{\mathrm{EtOH}: c l a y}$ equals to ca. $5 \times 10^{-2}$. This result is consistent with the composition range $\left(6 \times 10^{-3}<R_{\text {EtOH:clay }}\right.$ $<6 \times 10^{-2}$ ) in which the ethanol confinement occurs. The Knudsen thermogravimetric data (Fig. 6) allowed us to interpret the loading results (Fig. 1) by taking into account that the replacement of the ethanol solution inside the halloysite lumen is facilitated under low-pressure conditions. This finding is related to the enhancement of ethanol volatilization rate once the solvent vapor pressure is approaching. Consequently, the amounts of drug molecules entrapped into the cavity can be increased by vacuum pumping operation.
Fig. 5 Schematic representation for the effect of the loading conditions on the final features of the halloysite/drug composites

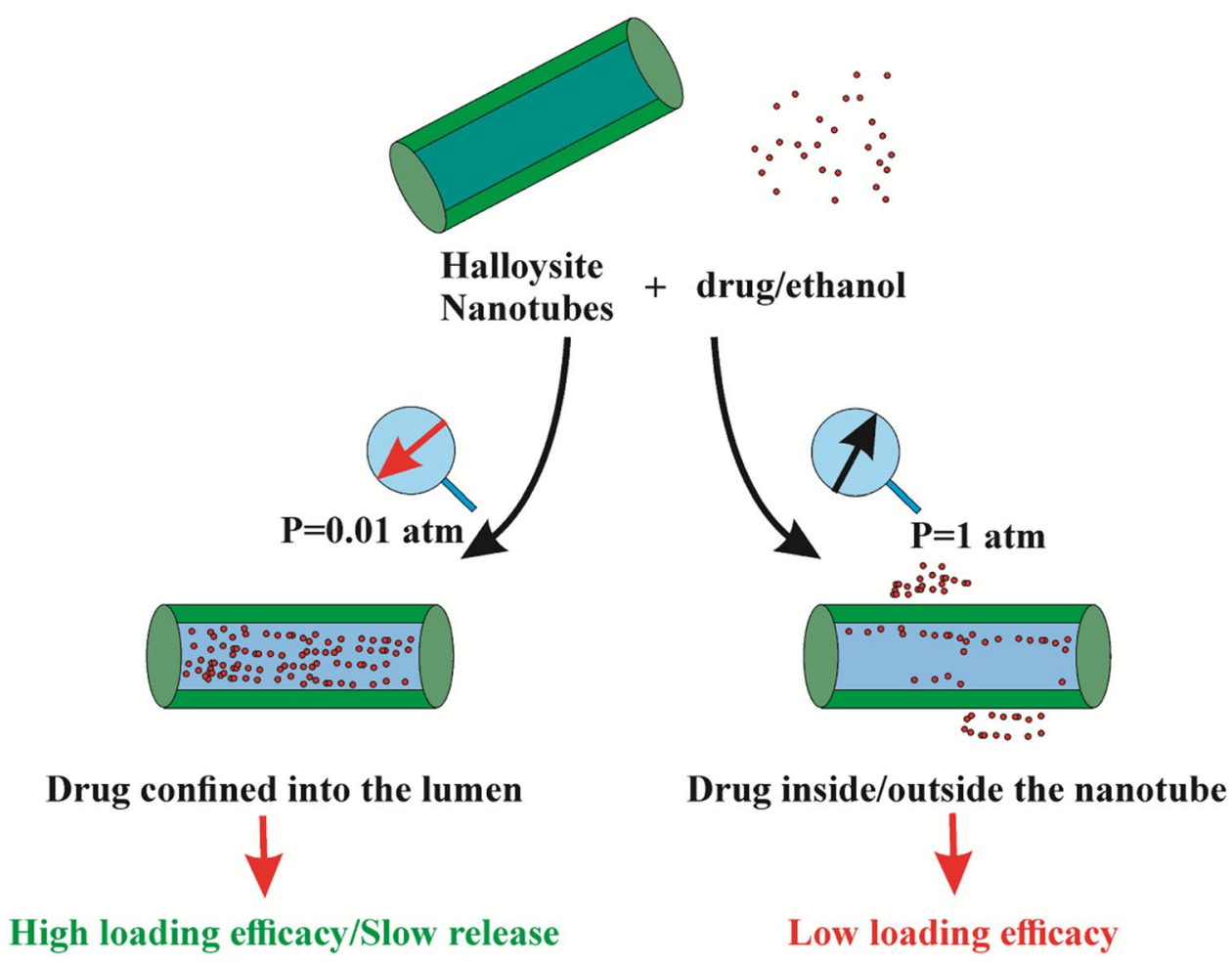

High loading efficacy/Slow release
Springer 


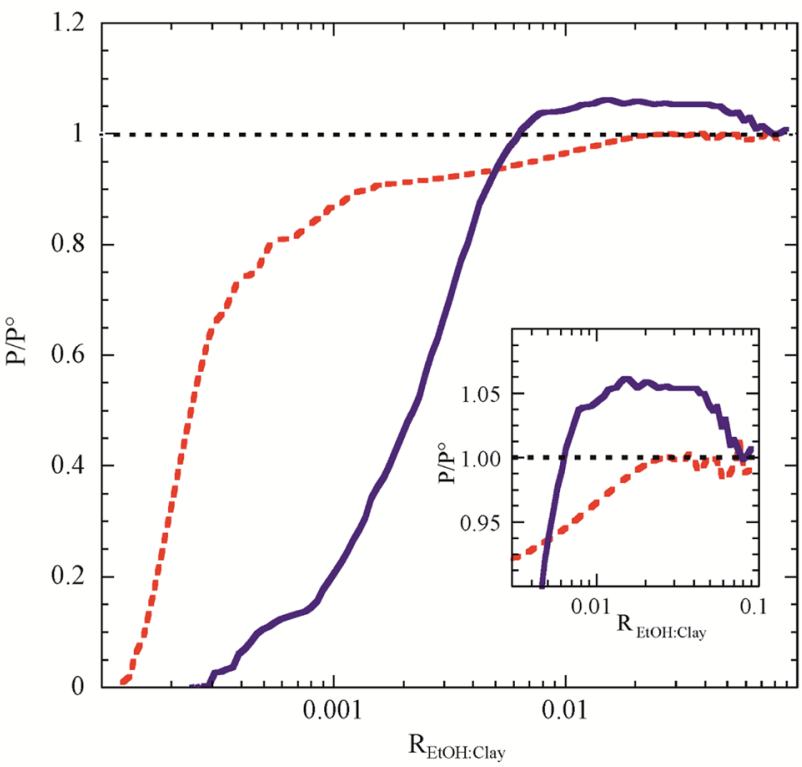

Fig. 6 Relative vapor pressure for ethanol as a function of the ethanol to clay mass ratio for kaolinite (red broken line) and halloysite (full blue line) system

Table 2 Parameters for the ethanol desorption isotherms

\begin{tabular}{lclr}
\hline & $R^{\circ}\left(g_{\text {ethanol }} / g_{\text {clay }}\right)$ & $K$ & \multicolumn{1}{l}{$C$} \\
\hline HNTs & $(1.80 \pm 0.09) \cdot 10^{-3}$ & $0.714 \pm 0.016$ & $5.7 \pm 0.6$ \\
Kaolinite & $(6.23 \pm 0.03) \cdot 10^{-5}$ & $1.008 \pm 0.001$ & $50.2 \pm 0.9$ \\
\hline
\end{tabular}

From the data measured in the low $R_{\mathrm{EtOH}: c l a y}$ range (corresponding to $P / P^{\circ}$ values between 0.05 and 0.95 ), one can investigate the ethanol isothermal desorption from the nanoclays surfaces. Similar to water/clay systems, the Guggenheim-Anderson-de Boer (GAB) model [76] can be used as fitting model, which is expressed by the following equation: than the corresponding constant for monolayer. This result indicates that the ethanol-ethanol interactions are favored compared to those between ethanol and nanoclays [77].

Despite the equivalent surface chemistry, the monolayer saturation was reached at different the ethanol:clay mass ratios for halloysite and kaolinite. In particular, halloysite can adsorb an amount of ethanol that is ca. 30 times larger than kaolinite. This result cannot be ascribed to the larger specific surface of HNT because the specific surface ratio between HNT and Kao is ca. 3.5 [66] and, therefore, it reflects a denser ethanol layer on the halloysite nanotubes than in flat kaolinite.

\section{Conclusions}

This work explores the filling of halloysite nanotubes with drug molecules (salicylic acid and sodium diclofenac) dissolved in ethanol solvent. The influence of the pressure conditions on the loading efficiencies and the release kinetics was investigated by thermodynamic and structural viewpoints. We observed that the vacuum pumping in/out operation determines an increase of the loading amounts of both drugs, which exhibited a slower release kinetics with respect to the corresponding loaded nanotubes prepared at environment pressure. These results were explained on the basis of the Knudsen thermogravimetry experiments, which proved the ethanol confinement within the halloysite cavity as a consequence of the Gibbs Thomson effect. Compared to the bulk solvent, the confined ethanol evidenced a faster evaporation rate that generates a flux of the drug solution within the nanotubes' cavity, and, consequently, a payload of the guest molecules. This process is enhanced at reduced pressure conditions driving to increase the filling efficiency of halloysite nanotubes as confirmed by morphological

$R_{\text {EtOH : clay }}=\left(R^{\circ} \times C \times K \times P / P^{\circ}\right) /\left[\left(1-K \times P / P^{\circ}\right) \cdot\left(1-K \times P / P^{\circ}+C \times K \times P / P^{\circ}\right)\right]$,

being $R^{\circ}$ the ethanol:clay mass ratio for the monolayer coverage, while $C$ is a constant related to the monolayer adsorption. Moreover, $K$ is a coefficient that depends on the formation of multilayers during the adsorption process.

It should be noted that the GAB model reveals successful in the fitting of ethanol/HNTs and ethanol/kaolinite desorption isotherms. As an example, Supplementary Material (Fig. 4S) shows the fitting by GAB model for the ethanol desorption from HNTs. The adsorption parameters obtained by GAB method are provided in Table 2 . Both clays provided $C>2$ and, therefore, the ethanol desorption isotherms can be classified as type III [76]. The equilibrium constant value for the multilayer formation is ca. one order of magnitude larger investigations. According to these considerations, the loadings and the release properties were not significantly affected by pressure conditions for platy kaolinite filled with salicylic acid and sodium diclofenac. It should be noted that the drugs encapsulation slightly affected the halloysite surface charge ruling out the presence of relevant electrostatic interactions between halloysite inner surface and the guest molecules.

The present work represents a fundamental step for development of halloysite-based nanocarriers with tunable drug loading and release kinetics.

Supplementary Information The online version contains supplementary material available at https://doi.org/10.1007/s40097-021-00391-z. 
Acknowledgements The work was financially supported by University of Palermo as well as by AGM for CuHe" (ARS01_00697) project.

Funding Open Access funding provided by Università degli Studi di Palermo.

\section{Compliance with ethical standards}

Conflict of interest The authors declare that there is no conflict of interest.

Open Access This article is licensed under a Creative Commons Attribution 4.0 International License, which permits use, sharing, adaptation, distribution and reproduction in any medium or format, as long as you give appropriate credit to the original author(s) and the source, provide a link to the Creative Commons licence, and indicate if changes were made. The images or other third party material in this article are included in the article's Creative Commons licence, unless indicated otherwise in a credit line to the material. If material is not included in the article's Creative Commons licence and your intended use is not permitted by statutory regulation or exceeds the permitted use, you will need to obtain permission directly from the copyright holder. To view a copy of this licence, visit http://creativecommons.org/licenses/by/4.0/.

\section{References}

1. Du, M., Guo, B., Jia, D.: Newly emerging applications of halloysite nanotubes: a review. Polym. Int. 59, 574-582 (2010)

2. Paineau, E., Krapf, M.-E.M., Amara, M.-S., Matskova, N.V., Dozov, I., Rouzière, S., Thill, A., Launois, P., Davidson, P.: A liquid-crystalline hexagonal columnar phase in highly-dilute suspensions of imogolite nanotubes. Nat. Commun. 7, 10271 (2016)

3. Suman, K., Joshi, Y.M.: Microstructure and soft glassy dynamics of an aqueous laponite dispersion. Langmuir 34, 13079-13103 (2018)

4. Rebitski, E.P., Aranda, P., Darder, M., Carraro, R., Ruiz-Hitzky, E.: Intercalation of metformin into montmorillonite. Dalton Trans. 47, 3185-3192 (2018)

5. Djellali, M., Aranda, P., Ruiz-Hitzky, E.: Silica-layered double hydroxide nanoarchitectured materials. Appl. Clay Sci. 171, 65-73 (2019)

6. Cheikh, D., García-Villén, F., Majdoub, H., Zayani, M.B., Viseras, C.: Complex of chitosan pectin and clay as diclofenac carrier. Appl. Clay Sci. 172, 155-164 (2019)

7. del Campo, M.M.G., Darder, M., Aranda, P., Akkari, M., Huttel, Y., Mayoral, A., Bettini, J., Ruiz-Hitzky, E.: Functional hybrid nanopaper by assembling nanofibers of cellulose and sepiolite. Adv. Funct. Mater. 28, 1703048 (2017)

8. Abdullayev, E., Lvov, Y.: Halloysite clay nanotubes as a ceramic "skeleton" for functional biopolymer composites with sustained drug release. J. Mater. Chem. B 1, 2894-2903 (2013)

9. Faustini, M., Nicole, L., Ruiz-Hitzky, E., Sanchez, C.: History of organic-inorganic hybrid materials: prehistory, art, science, and advanced applications. Adv. Funct. Mater. 28, 1704158 (2018)

10. Lee, S.S., Schmidt, M., Sturchio, N.C., Nagy, K.L., Fenter, P.: Effect of $\mathrm{pH}$ on the formation of gibbsite-layer films at the muscovite (001)-water interface. J. Phys. Chem. C 123, 6560-6571 (2019)

11. Attia, N.F., Mohamed, G.G., Ismail, M.M., Abdou, T.T.: Influence of organic modifier structures of 2D clay layers on thermal stability, flammability and mechanical properties of their rubber nanocomposites. J. Nanostructure Chem. 10, 161-168 (2020)
12. Pant, P., Bansal, R., Gulati, S., Kumar, S., Kodwani, R.: Porous and chelated nanostructured multifunctional materials: recoverable and reusable sorbents for extraction of metal ions and catalysts for diverse organic reactions. J. Nanostructure Chem. 6, 145-157 (2016)

13. Prasad, C., Tang, H., Liu, W.: Magnetic Fe3O4 based layered double hydroxides (LDHs) nanocomposites (Fe3O4/LDHs): recent review of progress in synthesis, properties and applications. J. Nanostructure Chem. 8, 393-412 (2018)

14. Bugatti, V., Vertuccio, L., Zara, S., Fancello, F., Scanu, B., Gorrasi, G.: Green pesticides based on cinnamate anion incorporated in layered double hydroxides and dispersed in pectin matrix. Carbohydr. Polym. 209, 356-362 (2019)

15. Zhang, J., Liu, T., Liu, M.: Hydrothermal synthesis of halloysite nanotubes@carbon nanocomposites with good biocompatibility. Microporous Mesoporous Mater. 266, 155-163 (2018)

16. Ghiassi, S., Sedaghat, S., Mokhtary, M., Kefayati, H.: Plant-mediated bio-synthesis of silver-montmorillonite nanocomposite and antibacterial effects on gram-positive and -negative bacteria. J. Nanostructure Chem. 8, 353-357 (2018)

17. Ouyang, J., Liu, T., Yang, H., Zhang, Y.: Multiple polarization loss and permittivity adjusting of halloysite/BN co-doped carbon/cobalt composites. J. Colloid Interface Sci. 555, 509-518 (2019)

18. Ferrante, F., Armata, N., Cavallaro, G., Lazzara, G.: Adsorption studies of molecules on the halloysite surfaces: a computational and experimental investigation. J. Phys. Chem. C 121, 2951-2958 (2017)

19. Zhang, Y., Bai, L., Cheng, C., Zhou, Q., Zhang, Z., Wu, Y., Zhang, H.: A novel surface modification method upon halloysite nanotubes: a desirable cross-linking agent to construct hydrogels. Appl. Clay Sci. 182, 105259 (2019)

20. Lvov, Y.M., Shchukin, D.G., Mohwald, H., Price, R.R.: Halloysite clay nanotubes for controlled release of protective agents. ACS Nano 2, 814-820 (2008)

21. Lazzara, G., Cavallaro, G., Panchal, A., Fakhrullin, R., Stavitskaya, A., Vinokurov, V., Lvov, Y.: An assembly of organicinorganic composites using halloysite clay nanotubes. Curr. Opin. Colloid Interface Sci. 35, 42-50 (2018)

22. Cavallaro, G., Chiappisi, L., Pasbakhsh, P., Gradzielski, M., Lazzara, G.: A structural comparison of halloysite nanotubes of different origin by small-angle neutron scattering (SANS) and electric birefringence. Appl. Clay Sci. 160, 71-80 (2018)

23. Liu, M., Huo, Z., Liu, T., Shen, Y., He, R., Zhou, C.: Selfassembling halloysite nanotubes into concentric ring patterns in a sphere-on-flat geometry. Langmuir 33, 3088-3098 (2017)

24. Emmanuel, E., Yong, L.L., Anggraini, V., Pasbakhsh, P.: Can halloysite nanotubes be used to remediate zinc and lead-contaminated marine clay? A solidification/stabilization approach. Appl. Clay Sci. 186, 105441 (2020)

25. Sadjadi, S., Heravi, M.M., Kazemi, S.S.: Ionic liquid decorated chitosan hybridized with clay: a novel support for immobilizing Pd nanoparticles. Carbohydr. Polym. 200, 183-190 (2018)

26. Lvov, Y., Panchal, A., Fu, Y., Fakhrullin, R., Kryuchkova, M., Batasheva, S., Stavitskaya, A., Glotov, A., Vinokurov, V.: Interfacial self-assembly in halloysite nanotube composites. Langmuir 35, 8646-8657 (2019)

27. Panchal, A., Fakhrullina, G., Fakhrullin, R., Lvov, Y.: Selfassembly of clay nanotubes on hair surface for medical and cosmetic formulations. Nanoscale 10, 18205-18216 (2018)

28. Zhang, H., Cheng, C., Song, H., Bai, L., Cheng, Y., Ba, X., Wu, Y.: A facile one-step grafting of polyphosphonium onto halloysite nanotubes initiated by $\mathrm{Ce}(\mathrm{IV})$. Chem. Commun. 55, 1040-1043 (2019)

29. Cheng, C., Gao, Y., Song, W., Zhao, Q., Zhang, H., Zhang, $\mathrm{H}$.: Halloysite nanotube-based $\mathrm{H} 2 \mathrm{O} 2$-responsive drug delivery 
system with a turn on effect on fluorescence for real-time monitoring. Chem. Eng. J. 380, 122474 (2020)

30. Liu, F., Bai, L., Zhang, H., Song, H., Hu, L., Wu, Y., Ba, X.: Smart H2O2-responsive drug delivery system made by halloysite nanotubes and carbohydrate polymers. ACS Appl. Mater. Interfaces 9, 31626-31633 (2017)

31. Zhao, Y., Kong, W., Jin, Z., Fu, Y., Wang, W., Zhang, Y., Liu, J., Zhang, B.: Storing solar energy within Ag-Paraffin@Halloysite microspheres as a novel self-heating catalyst. Appl. Energy 222, $180-188$ (2018)

32. Zhao, X., Wan, Q., Fu, X., Meng, X., Ou, X., Zhong, R., Zhou, Q., Liu, M.: Toxicity evaluation of one-dimensional nanoparticles using caenorhabditis elegans: a comparative study of halloysite nanotubes and chitin nanocrystals. ACS Sustain. Chem. Eng. 7, 18965-18975 (2019)

33. Wu, F., Pickett, K., Panchal, A., Liu, M., Lvov, Y.: Superhydrophobic polyurethane foam coated with polysiloxane-modified clay nanotubes for efficient and recyclable oil absorption. ACS Appl. Mater. Interfaces 11, 25445-25456 (2019)

34. Cavallaro, G., Grillo, I., Gradzielski, M., Lazzara, G.: Structure of hybrid materials based on halloysite nanotubes filled with anionic surfactants. J. Phys. Chem. C 120, 13492-13502 (2016)

35. Sadjadi, S., Malmir, M., Heravi, M.M., Kahangi, F.G.: Biocompatible starch-halloysite hybrid: an efficient support for immobilizing Pd species and developing a heterogeneous catalyst for ligand and copper free coupling reactions. Int. J. Biol. Macromol. 118, 1903-1911 (2018)

36. Taroni, T., Meroni, D., Fidecka, K., Maggioni, D., Longhi, M., Ardizzone, S.: Halloysite nanotubes functionalization with phosphonic acids: Role of surface charge on molecule localization and reversibility. Appl. Surf. Sci. 486, 466-473 (2019)

37. Bertolino, V., Cavallaro, G., Lazzara, G., Milioto, S., Parisi, F.: Biopolymer-targeted adsorption onto halloysite nanotubes in aqueous media. Langmuir 33, 3317-3323 (2017)

38. Cavallaro, G., Milioto, S., Konnova, S., Fakhrullina, G., Akhatova, F., Lazzara, G., Fakhrullin, R., Lvov, Y.: Halloysite/keratin nanocomposite for human hair photoprotection coating. ACS Appl. Mater. Interfaces 12, 24348-24362 (2020)

39. Ojo, O.F., Farinmade, A., Trout, J., Omarova, M., He, J., John, V., Blake, D.A., Lvov, Y.M., Zhang, D., Nguyen, D., Bose, A.: Stoppers and skins on clay nanotubes help stabilize oil-in-water emulsions and modulate the release of encapsulated surfactants. ACS Appl. Nano Mater. 2, 3490-3500 (2019)

40. Yendluri, R., Lvov, Y., de Villiers, M.M., Vinokurov, V., Naumenko, E., Tarasova, E., Fakhrullin, R.: Paclitaxel encapsulated in halloysite clay nanotubes for intestinal and intracellular delivery. J. Pharm. Sci. 106, 3131-3139 (2017)

41. Wu, Y.-P., Yang, J., Gao, H.-Y., Shen, Y., Jiang, L., Zhou, C., Li, Y.-F., He, R.-R., Liu, M.: Folate-conjugated halloysite nanotubes, an efficient drug carrier, deliver doxorubicin for targeted therapy of breast cancer. ACS Appl. Nano Mater. 1, 595-608 (2018)

42. Bugatti, V., Viscusi, G., Naddeo, C., Gorrasi, G.: Nanocomposites based on PCL and halloysite nanotubes filled with lysozyme: effect of draw ratio on the physical properties and release analysis. Nanomaterials 7, 213 (2017)

43. Owoseni, O., Nyankson, E., Zhang, Y., Adams, S.J., He, J., McPherson, G.L., Bose, A., Gupta, R.B., John, V.T.: Release of surfactant cargo from interfacially-active halloysite clay nanotubes for oil spill remediation. Langmuir 30, 13533-13541 (2014)

44. Tan, D., Yuan, P., Annabi-Bergaya, F., Yu, H., Liu, D., Liu, H., He, H.: Natural halloysite nanotubes as mesoporous carriers for the loading of ibuprofen. Microporous Mesoporous Mater. 179, 89-98 (2013)
45. Jiang, W.-T., Chang, P.-H., Tsai, Y., Li, Z.: Halloysite nanotubes as a carrier for the uptake of selected pharmaceuticals. Microporous Mesoporous Mater. 220, 298-307 (2016)

46. Fakhrullina, G.I., Akhatova, F.S., Lvov, Y.M., Fakhrullin, R.F.: Toxicity of halloysite clay nanotubes in vivo: a Caenorhabditis elegans study. Environ. Sci. Nano 2, 54-59 (2015)

47. Santos, A.C., Ferreira, C., Veiga, F., Ribeiro, A.J., Panchal, A., Lvov, Y., Agarwal, A.: Halloysite clay nanotubes for life sciences applications: from drug encapsulation to bioscaffold. Adv. Colloid Interface Sci. 257, 58-70 (2018)

48. Cavallaro, G., Lazzara, G., Lisuzzo, L., Milioto, S., Parisi, F.: Selective adsorption of oppositely charged PNIPAAM on halloysite surfaces: a route to thermo-responsive nanocarriers. Nanotechnology 29, 325702 (2018)

49. Lisuzzo, L., Cavallaro, G., Milioto, S., Lazzara, G.: Layered composite based on halloysite and natural polymers: a carrier for the pH controlled release of drugs. New J. Chem. 43, 10887-10893 (2019)

50. Aguzzi, C., Viseras, C., Cerezo, P., Salcedo, I., Sánchez-Espejo, R., Valenzuela, C.: Release kinetics of 5-aminosalicylic acid from halloysite. Colloids Surf. B Biointerfaces 105, 75-80 (2013)

51. Lisuzzo, L., Wicklein, B., Dico, G.L., Lazzara, G., del Real, G., Aranda, P., Ruiz-Hitzky, E.: Functional biohybrid materials based on halloysite, sepiolite and cellulose nanofibers for health applications. Dalton Trans. 49, 3830-3840 (2020)

52. Čalija, B., Milić, J., Milašinović, N., Daković, A., Trifković, K., Stojanović, J., Krajišnik, D.: Functionality of chitosan-halloysite nanocomposite films for sustained delivery of antibiotics: the effect of chitosan molar mass. J. Appl. Polym. Sci. 137, 48406 (2020)

53. Abdullayev, E., Lvov, Y.: Clay nanotubes for corrosion inhibitor encapsulation: release control with end stoppers. J Mater Chem. 20, 6681-6687 (2010)

54. Joshi, A., Abdullayev, E., Vasiliev, A., Volkova, O., Lvov, Y.: Interfacial modification of clay nanotubes for the sustained release of corrosion inhibitors. Langmuir 29, 7439-7448 (2013)

55. Abdullayev, E., Price, R., Shchukin, D., Lvov, Y.: Halloysite tubes as nanocontainers for anticorrosion coating with benzotriazole. ACS Appl. Mater. Interfaces 1, 1437-1443 (2009)

56. Makaremi, M., Pasbakhsh, P., Cavallaro, G., Lazzara, G., Aw, Y.K., Lee, S.M., Milioto, S.: Effect of morphology and size of halloysite nanotubes on functional pectin bionanocomposites for food packaging applications. ACS Appl. Mater. Interfaces 9, 17476-17488 (2017)

57. Cavallaro, G., Milioto, S., Parisi, F., Lazzara, G.: Halloysite nanotubes loaded with calcium hydroxide: alkaline fillers for the deacidification of waterlogged archeological woods. ACS Appl. Mater. Interfaces 10, 27355-27364 (2018)

58. Rawtani, D., Pandey, G., Tharmavaram, M., Pathak, P., Akkireddy, S., Agrawal, Y.K.: Development of a novel 'nanocarrier' system based on halloysite nanotubes to overcome the complexation of ciprofloxacin with iron: an in vitro approach. Appl. Clay Sci. 150, 293-302 (2017)

59. Pandey, G., Munguambe, D.M., Tharmavaram, M., Rawtani, D., Agrawal, Y.K.: Halloysite nanotubes - an efficient 'nano-support' for the immobilization of $\alpha$-amylase. Appl. Clay Sci. 136, 184191 (2017)

60. Saadat, S., Pandey, G., Tharmavaram, M., Braganza, V., Rawtani, D.: Nano-interfacial decoration of halloysite nanotubes for the development of antimicrobial nanocomposites. Adv. Colloid Interface Sci. 275, 102063 (2020)

61. Tharmavaram, M., Pandey, G., Rawtani, D.: Surface modified halloysite nanotubes: a flexible interface for biological, environmental and catalytic applications. Adv. Colloid Interface Sci. 261, 82-101 (2018) 
62. Barman, M., Mahmood, S., Augustine, R., Hasan, A., Thomas, S., Ghosal, K.: Natural halloysite nanotubes /chitosan based bionanocomposite for delivering norfloxacin, an anti-microbial agent in sustained release manner. Int. J. Biol. Macromol. 162, 18491861 (2020)

63. Cavallaro, G., Lazzara, G., Milioto, S., Parisi, F., Evtugyn, V., Rozhina, E., Fakhrullin, R.: Nanohydrogel formation within the halloysite lumen for triggered and sustained release. ACS Appl. Mater. Interfaces 10, 8265-8273 (2018)

64. Kim, B.M., Qian, S., Bau, H.H.: Filling carbon nanotubes with particles. Nano Lett. 5, 873-878 (2005)

65. Price, R.R., Gaber, B.P., Lvov, Y.: In vitro release characteristics of tetracyline $\mathrm{HCl}$, khellin and nicotinamide adenine dinucloetide from halloysite; a cylindrical mineral. J Microencapsul. 18, 713 (2001)

66. Lisuzzo, L., Cavallaro, G., Pasbakhsh, P., Milioto, S., Lazzara, G.: Why does vacuum drive to the loading of halloysite nanotubes? The key role of water confinement. J. Colloid Interface Sci. 547, 361-369 (2019)

67. Farinmade, A., Ojo, O.F., Trout, J., He, J., John, V., Blake, D.A., Lvov, Y.M., Zhang, D., Nguyen, D., Bose, A.: Targeted and stimulus-responsive delivery of surfactant to the oil-water interface for applications in oil spill remediation. ACS Appl. Mater. Interfaces 12, 1840-1849 (2020)

68. Golubeva, O.Y., Alikina, Y.A., Brazovskaya, E.Y., Ugolkov, V.V.: Peculiarities of the 5-fluorouracil adsorption on porous aluminosilicates with different morphologies. Appl. Clay Sci. 184, 105401 (2020)

69. Shalmashi, A., Eliassi, A.: Solubility of salicylic acid in water, ethanol, carbon tetrachloride, ethyl acetate, and xylene. J. Chem. Eng. Data 53, 199-200 (2008)

70. Saei, A.A., Jabbaribar, F., Fakhree, M.A.A., Acree, W.E., Jouyban, A.: Solubility of sodium diclofenac in binary water + alcohol solvent mixtures at $25^{\circ}$ C. J. Drug Deliv. Sci. Technol. 18, 149-151 (2008)
71. Yu, D., Wang, J., Hu, W., Guo, R.: Preparation and controlled release behavior of halloysite/2-mercaptobenzothiazole nanocomposite with calcined halloysite as nanocontainer. Mater. Des. 129, 103-110 (2017)

72. Blanco, I., Abate, L., Bottino, F.A., Bottino, P.: Thermal behaviour of a series of novel aliphatic bridged polyhedral oligomeric silsesquioxanes (POSSs)/polystyrene (PS) nanocomposites: the influence of the bridge length on the resistance to thermal degradation. Polym. Degrad. Stab. 102, 132-137 (2014). https://doi. org/10.1016/j.polymdegradstab.2014.01.029

73. Massaro, M., Cavallaro, G., Colletti, C.G., D’Azzo, G., Guernelli, S., Lazzara, G., Pieraccini, S., Riela, S.: Halloysite nanotubes for efficient loading, stabilization and controlled release of insulin. J. Colloid Interface Sci. 524, 156-164 (2018)

74. Schneider, C.A., Rasband, W.S., Eliceiri, K.W.: NIH Image to ImageJ: 25 years of image analysis. Nat. Methods 9, 671-675 (2012)

75. Lisuzzo, L., Cavallaro, G., Parisi, F., Milioto, S., Fakhrullin, R., Lazzara, G.: Core/shell gel beads with embedded halloysite nanotubes for controlled drug release. Coatings 9, 70 (2019)

76. Fotie, G., Rampazzo, R., Ortenzi, M.A., Checchia, S., Fessas, D., Piergiovanni, L.: The effect of moisture on cellulose nanocrystals intended as a high gas barrier coating on flexible packaging materials. Polymers 9, 415 (2017)

77. Yogendrarajah, P., Samapundo, S., Devlieghere, F., De Saeger, S., De Meulenaer, B.: Moisture sorption isotherms and thermodynamic properties of whole black peppercorns (Piper nigrum L.). LWT Food Sci. Technol. 64, 177-188 (2015)

Publisher's Note Springer Nature remains neutral with regard to jurisdictional claims in published maps and institutional affiliations.

\title{
Authors and Affiliations
}

\author{
Lorenzo Lisuzzo $^{1} \cdot$ Giuseppe Cavallaro ${ }^{1,2} \mathbb{D} \cdot$ Stefana Milioto ${ }^{1,2} \cdot$ Giuseppe Lazzara $^{1,2}$ \\ 1 Dipartimento di Fisica e Chimica "Emilio Segrè", Università \\ degli Studi di Palermo, Viale delle Scienze, pad. 17, \\ 90128 Palermo, Italy \\ 2 Consorzio Interuniversitario Nazionale per la Scienza \\ e Tecnologia dei Materiali, INSTM, Via G. Giusti, 9, \\ 50121 Florence, Italy
}

